PROCEEDINGS OF THE

AMERICAN MATHEMATICAL SOCIETY

Volume 135, Number 11, November 2007, Pages 3425-3433

S 0002-9939(07)08957-5

Article electronically published on August 6, 2007

\title{
ON IGUSA ZETA FUNCTIONS OF MONOMIAL IDEALS
}

\author{
JASON HOWALD, MIRCEA MUSTAŢĂ, AND CORNELIA YUEN
}

(Communicated by Bernd Ulrich)

\begin{abstract}
We show that the real parts of the poles of the Igusa zeta function of a monomial ideal can be computed from the torus-invariant divisors on the normalized blow-up of the affine space along the ideal. Moreover, we show that every such number is a root of the Bernstein-Sato polynomial associated to the monomial ideal.
\end{abstract}

\section{INTRODUCTION}

If $f$ is a nonconstant polynomial in $\mathbb{Z}\left[x_{1}, \ldots, x_{n}\right]$ and $p$ is a fixed prime, then the Igusa zeta function of $f$ is defined by

$$
Z_{f}(s)=\int_{\left(\mathbb{Z}_{p}\right)^{n}}|f(y)|_{p}^{s}|d y|,
$$

for every $s \in \mathbb{C}$ with $\operatorname{Re}(s)>0$. Here $\mathbb{Z}_{p}$ denotes the ring of $p$-adic integers, endowed with the discrete valuation $\operatorname{ord}_{p}$ and with the $p$-adic absolute value defined by

$$
|a|_{p}=\left(\frac{1}{p}\right)^{\operatorname{ord}_{p}(a)} \text {. }
$$

The measure $\mu$ on $\left(\mathbb{Z}_{p}\right)^{n}$ that is used in the above integral is the Haar measure characterized by

$$
\mu\left(\prod_{i=1}^{n} p^{m_{i}} \mathbb{Z}_{p}\right)=\left(\frac{1}{p}\right)^{\sum_{i} m_{i}}
$$

As defined, $Z_{f}$ is a holomorphic function on the half plane $\{s \mid \operatorname{Re}(s)>0\}$, and one can show that it admits a meromorphic extension to $\mathbb{C}$. In fact, $Z_{f}$ is a rational function of $\left(\frac{1}{p}\right)^{s}$. A proof of rationality that also gives information on the real parts of the possible poles of $Z_{f}$ proceeds as follows. Let $\pi: Y \rightarrow X=\mathbb{A}^{n}$ be an embedded resolution of singularities of $f$ defined over $\mathbb{Q}$. This means that $\pi$ is proper and birational, $Y$ is smooth, and the union of $\pi^{*}(\operatorname{div}(f))$ and of the exceptional locus of $\pi$ is a divisor with simple normal crossings. For every prime divisor $E$ in this union, denote by $a_{E}(f)$ the order of $E$ in $\pi^{*}(\operatorname{div}(f))$ and by $k_{E}$ the order of $E$ in the relative canonical class $K_{Y / X}$ (this is the divisor locally defined by $\operatorname{det}(\operatorname{Jac}(\pi)))$. Using the Change of Variable formula for $p$-adic integrals to express

Received by the editors June 15, 2006.

2000 Mathematics Subject Classification. Primary 14B05; Secondary 14M25.

Key words and phrases. Igusa zeta function, monomial ideal, Bernstein-Sato polynomial.

The research of the second author was partially supported by NSF grant DMS 0500127.

(C)2007 American Mathematical Society Reverts to public domain 28 years from publication 
$Z_{f}$ as an integral over $Y\left(\mathbb{Z}_{p}\right)$, Igusa obtained the rationality of $Z_{f}$ as a function of $\left(\frac{1}{p}\right)^{s}$ and the fact that if $s$ is a pole of $Z_{f}$, then $\operatorname{Re}(s)=-\frac{k_{E}+1}{a_{E}(f)}$ for some divisor $E$ as above. Our main reference for Igusa zeta functions is Igusa's book Ig] (see also Denef's Bourbaki report [De]).

While every divisor on a log resolution of $f$ gives a candidate for the real part of a pole of $Z_{f}$, examples show that most of these numbers do not come actually from poles of $Z_{f}$. In fact an outstanding open problem in the field is the following conjecture of Igusa, relating the poles to one of the basic invariants of the singularities of $f$, its Bernstein-Sato polynomial.

Conjecture 1.1. Let $f$ be a nonconstant polynomial in $\mathbb{Z}\left[X_{1}, \ldots, X_{n}\right]$. For almost all primes $p$ the following holds: if $s$ is a pole of $Z_{f}$, then the real part of $s$ is a root of the Bernstein-Sato polynomial $b_{f}$ of $f$.

We recall that the Bernstein-Sato polynomial of $f$ is a polynomial in one variable introduced independently in $[\mathrm{Be}$ and $[\mathrm{SS}]$. It is a subtle but very fundamental invariant of the singularities of $f$. We do not give its definition as we will not need it, but we mention that its roots are related to the eigenvalues of the monodromy of the hypersurface $f^{-1}(0)$. There is, in fact, a weaker version of the above conjecture that is stated in terms of these eigenvalues and that is known as the Monodromy Conjecture (see [De for more on these conjectures and also $\mathrm{Ve}$ ] for some recent work in this direction).

Our goal in this note is to prove the analogue of Conjecture 1.1 when we replace $f$ by a monomial ideal. Though less studied, Igusa zeta functions for nonnecessarily principal ideals in $\mathbb{Z}\left[x_{1}, \ldots, x_{n}\right]$ can be defined in a very similar way with (11). More precisely, if $I$ is a nonzero proper ideal of $\mathbb{Z}\left[x_{1}, \ldots, x_{n}\right]$ and if we put for $y \in\left(\mathbb{Z}_{p}\right)^{n}$

$$
\operatorname{ord}_{p} I(y)=\min \left\{\operatorname{ord}_{p}(f(y)) \mid f \in I\right\},
$$

then we have

$$
Z_{I}(s)=\int_{\left(\mathbb{Z}_{p}\right)^{n}}\left(\frac{1}{p}\right)^{\operatorname{ord}_{p} I(y)}|d y| .
$$

The above-mentioned results in the case of one polynomial extend in a straightforward way to the case of an arbitrary ideal. Note that in order to prove rationality, we need to consider a $\log$ resolution of $I$ : this is a morphism $\pi: Y \rightarrow \mathbb{A}^{n}$ as before, such that $\pi^{-1}(V(I))$ is a Cartier divisor and its union with the exceptional locus of $\pi$ is a divisor with simple normal crossings. If $E$ is a prime divisor on $Y$ contained in this union, then $a_{E}(I)$ is by definition the coefficient of $E$ in $\pi^{-1}(V(I))$. As in the case of a principal ideal, one can show that given a $\log$ resolution $\pi$, for every pole $s$ of $Z_{I}$ there is a divisor $E$ on $Y$ such that $\operatorname{Re}(s)=-\frac{k_{E}+1}{a_{E}(I)}$.

On the other hand, the definition of the Bernstein-Sato polynomial has been extended in BMS3 from the case of one polynomial to that of an arbitrary ideal. This is again a polynomial in one variable, and therefore the analogue of Conjecture 1.1 makes sense in this case. We will prove the monomial case, i.e. when $I$ is generated by monomials.

Theorem 1.2. If $I$ is a nonzero proper monomial ideal of $\mathbb{Z}\left[x_{1}, \ldots, x_{n}\right]$, then for every prime $p$ and every pole $s$ of $Z_{I}$, the real part of $s$ is a root of the BernsteinSato polynomial of $I$. 
The key ingredient in the proof of the above theorem is a result on the poles of Igusa-type zeta functions associated to cones. Suppose that $N \simeq \mathbb{Z}^{n}$ is a lattice and that $\sigma$ is a pointed, rational, polyhedral cone in $N_{\mathbb{R}}=N \otimes_{\mathbb{Z}} \mathbb{R}$. We denote by $\sigma^{\circ}$ the relative interior of the cone $\sigma$. If $\sigma^{\vee}$ is the dual cone in $M_{\mathbb{R}}$, where $M=\operatorname{Hom}_{\mathbb{Z}}(N, \mathbb{Z})$, and if $\ell_{1}, \ell_{2}$ are elements in $\sigma^{\vee} \cap M$ such that $\sigma \cap\left\{v \mid \ell_{2}(v)=0\right\}=\{0\}$, then we put

$$
Z_{\sigma, \ell_{1}, \ell_{2}}(s):=\sum_{v \in \sigma^{\circ} \cap N}\left(\frac{1}{p}\right)^{\ell_{1}(v) s+\ell_{2}(v)} .
$$

It is easy to see (and it will follow from our computations) that this is well-defined and holomorphic in $\{s \in \mathbb{C} \mid \operatorname{Re}(s)>0\}$. We prove the following

Theorem 1.3. For every $\sigma, \ell_{1}$ and $\ell_{2}$ as above, $Z_{\sigma, \ell_{1}, \ell_{2}}$ is a rational function of $\left(\frac{1}{p}\right)^{s}$, and therefore can be meromorphically extended to $\mathbb{C}$. Moreover, for every pole $s$ of $Z_{\sigma, \ell_{1}, \ell_{2}}$ there is a primitive generator $v$ of a ray of $\sigma$ such that $\operatorname{Re}(s)=-\frac{\ell_{2}(v)}{\ell_{1}(v)}$.

Given a monomial ideal $I$, we give in the next section a formula for $Z_{I}$ in terms of suitable zeta functions for the cones in the normal fan to the Newton polyhedron of $I$ (we refer for the relevant definition and for the precise formula to that section). Let us just mention that this fan defines the toric variety that is the normalized blowing-up of $\mathbb{A}^{n}$ along the ideal $I$. Using this formula and Theorem 1.3. we will show in $\S 3$ that the real part of every pole of $Z_{I}$ corresponds to a torus-invariant divisor in the normalized blow-up of $\mathbb{A}^{n}$ along $I$ (despite the fact that the normalized blowing-up is not a log resolution of $I$ ).

Theorem 1.4. Let $I$ be a nonzero proper monomial ideal of $\mathbb{Z}\left[x_{1}, \ldots, x_{n}\right]$. For every pole $s$ of $Z_{I}$, there is a torus-invariant divisor $E$ on the normalized blowingup of $\mathbb{A}^{n}$ along I such that

$$
\operatorname{Re}(s)=-\frac{k_{E}+1}{a_{E}(I)} .
$$

On the other hand, explicit descriptions of the roots of the Bernstein-Sato polynomial of a monomial ideal have been obtained in BMS1] and BMS2]. We use the description in BMS2] and Theorem 1.4 to prove Theorem 1.2 in the last section.

We mention that a description for the Igusa zeta function of a monomial ideal has also been obtained by Zúñiga-Galindo in [Zu]. Moreover, similar results appear in the work of Denef and Hoornert [DH], in which one describes the poles of the Igusa zeta functions for nondegenerate hypersurfaces with respect to their Newton polyhedron. It is shown in loc. cit. that for such $f$ the real part of essentially any pole corresponds to a facet of the Newton polyhedron of $f$, as above. Moreover, Loeser [Lo] showed that under some mild extra assumptions these numbers are roots of the Bernstein-Sato polynomial of $f$, thus proving Conjecture 1.1 for such nondegenerate hypersurfaces. On the other hand, note that the relations between the respective Igusa zeta functions and between the Bernstein-Sato polynomials of $f$ and of the corresponding monomial ideal are not clear in general.

\section{Igusa ZETA FUnCTIONS OF MONOMIAL IDEALS}

Let $I$ be a nonzero proper ideal of $\mathbb{Z}\left[x_{1}, \ldots, x_{n}\right]$ generated by monomials. If $u=\left(u_{1}, \ldots, u_{n}\right) \in \mathbb{N}^{n}$, we denote by $x^{u}$ the corresponding monomial $x_{1}^{u_{1}} \ldots x_{n}^{u_{n}}$. 
The Newton polyhedron $P_{I}$ of $I$ is the convex hull of those $u$ in $\mathbb{N}^{n}$ such that $x^{u}$ is in $I$.

If $N=\mathbb{Z}^{n}$ and $N_{\mathbb{R}}=N \otimes_{\mathbb{Z}} \mathbb{R}$, we think of $P_{I}$ as lying in $M_{\mathbb{R}}$, where $M=$ $\operatorname{Hom}_{\mathbb{Z}}(N, \mathbb{Z})$. We denote by $\langle\cdot, \cdot\rangle$ the canonical pairing between $M$ and $N$. If we consider in $N_{\mathbb{R}}$ the cone generated by the elements of the standard basis $e_{1}, \ldots, e_{n}$, then the corresponding toric variety is the affine space $\mathbb{A}^{n}$ and the subscheme $V(I)$ is invariant under the torus action (we refer to $[\mathrm{Fu}$ for the basic notions on toric varieties). Hence the normalized blowing-up of $\mathbb{A}^{n}$ along $I$ is again a toric variety, and therefore it corresponds to a fan subdividing the above cone. This is the normal fan to the polyhedron $P_{I}$, that we will denote by $\Delta_{I}$. It is defined as follows: to each face $Q$ of $P_{I}$ one associates the cone

$$
\sigma_{Q}:=\left\{v \in N_{\mathbb{R}} \mid\langle u, v\rangle \leq\left\langle u^{\prime}, v\right\rangle \text { for every } u \in Q \text { and } u^{\prime} \in P_{I}\right\} .
$$

The fan $\Delta_{I}$ consists of the cones $\sigma_{Q}$, when $Q$ varies over the faces of $P_{I}$. Note that $\operatorname{dim}\left(\sigma_{Q}\right)=n-\operatorname{dim}(Q)$, so the rays of $\Delta_{I}$ correspond to the facets of $P_{I}$, and the maximal cones of $\Delta_{I}$ correspond to the vertices of $P_{I}$.

Let $p$ be a fixed prime. We now proceed to the computation of $Z_{I}$. For every $a=\left(a_{1}, \ldots, a_{n}\right) \in \mathbb{N}^{n}$ we consider the set $C_{a}=\prod_{i=1}^{n}\left(p^{a_{i}} \mathbb{Z}_{p} \backslash p^{a_{i}+1} \mathbb{Z}_{p}\right)$. Since each $p^{a_{i}} \mathbb{Z}_{p} \backslash p^{a_{i}+1} \mathbb{Z}_{p}$ is a disjoint union of $(p-1)$ translates of $p^{a_{i}+1} \mathbb{Z}_{p}$, we see that

$$
\mu\left(C_{a}\right)=(p-1)^{n}\left(\frac{1}{p}\right)^{n+\sum_{i} a_{i}} .
$$

We denote by $e$ the vector $(1, \ldots, 1)$, so $\langle e, a\rangle=\sum_{i} a_{i}$.

The function $\operatorname{ord}_{p} I$ is constant on $C_{a}$ with value

$$
\nu(a):=\min \left\{\langle u, a\rangle \mid x^{u} \in I\right\}=\min \left\{\langle u, a\rangle \mid u \in P_{I}\right\} .
$$

Since the sets $C_{a}$ are disjoint and the complement of their union has measure zero, we deduce

$$
Z_{I}(s)=\sum_{a \in \mathbb{N}^{n}}\left(1-\frac{1}{p}\right)^{n} \cdot\left(\frac{1}{p}\right)^{\langle e, a\rangle+s \nu(a)} .
$$

Note that $\nu$ is a linear function on each of the cones in $\Delta_{I}$. Indeed, if $w$ is a vertex of $P_{I}$, then $\nu(a)=\langle w, a\rangle$ whenever $a$ is in $\sigma_{w}$.

If $\sigma$ is a cone in $\Delta_{I}$, choose a vertex $w$ of $P_{I}$ such that $\sigma$ is contained in $\sigma_{w}$ and put $\ell_{\sigma}:=w$. By letting the $a$ in (4) vary inside the relative interior of each cone in $\Delta_{I}$, and using the definition in the Introduction, we get the following

Proposition 2.1. With the above notation, we have

$$
Z_{I}(s)=\left(1-\frac{1}{p}\right)^{n} \cdot \sum_{\sigma \in \Delta_{I}} Z_{\sigma, \ell_{\sigma}, e}(s)
$$

\section{IgUSA ZETA FUnCTIONS FOR CONES}

Our goal in this section is to discuss Igusa-type zeta functions associated to cones and prove Theorem 1.3. Let $N$ be a lattice, $M$ its dual, and $\sigma$ a pointed, rational polyhedral cone in $N_{\mathbb{R}}$. We consider $\ell_{1}$ and $\ell_{2}$ in $\sigma^{\vee} \cap M$, where $\sigma^{\vee}$ is the dual cone of $\sigma$, such that $\sigma \cap\left\{v \mid \ell_{2}(v)=0\right\}=\{0\}$. We want to study the function $Z_{\sigma, \ell_{1}, \ell_{2}}$ and its poles. 
The definition of $Z_{\sigma, \ell_{1}, \ell_{2}}$ was motivated by the formula in Proposition 2.1, but sometimes it is more natural to consider a version of this function in which we sum over all the integer points in $\sigma$ :

$$
\bar{Z}_{\sigma, \ell_{1}, \ell_{2}}(s):=\sum_{v \in \sigma \cap N}\left(\frac{1}{p}\right)^{\ell_{1}(v) s+\ell_{2}(v)} .
$$

Again, this is well-defined if $\operatorname{Re}(s)>0$ and we have $\bar{Z}_{\sigma, \ell_{1}, \ell_{2}}=\sum_{\tau} Z_{\tau, \ell_{1}, \ell_{2}}$, where the sum is over all faces $\tau$ of $\sigma$. Moreover, it follows from this formula that $Z_{\sigma, \ell_{1}, \ell_{2}}$ can be computed in terms of the functions $\bar{Z}_{\tau, \ell_{1}, \ell_{2}}$, where $\tau$ varies over the faces of $\sigma$.

We start with the following

Lemma 3.1. Let $v_{1}, \ldots, v_{r}$ in $N$ be linearly independent over $\mathbb{Q}$. If $w$ is in $N$ and $\ell_{1}, \ell_{2}$ are elements in $M$, with $\ell_{1}$ nonnegative and $\ell_{2}$ positive on all the $v_{i}$, then we put

$$
A(s):=\sum_{v \in S}\left(\frac{1}{p}\right)^{\ell_{1}(v) s+\ell_{2}(v)},
$$

where $S=\left\{w+a_{1} v_{1}+\ldots+a_{r} v_{r} \mid a=\left(a_{i}\right) \in \mathbb{N}^{r}\right\}$. The function $A$ is well-defined and holomorphic for $\operatorname{Re}(s)>0$ and it is a rational function in $\left(\frac{1}{p}\right)^{s}$, so it has a meromorphic continuation to $\mathbb{C}$. Moreover, if $s$ is a pole of $A$, then there is $i$ such that $\operatorname{Re}(s)=-\frac{\ell_{2}\left(v_{i}\right)}{\ell_{1}\left(v_{i}\right)}$.

Proof. If $\operatorname{Re}(s)>-\frac{\ell_{2}\left(v_{i}\right)}{\ell_{1}\left(v_{i}\right)}$ for all $i$ such that $\ell_{1}\left(v_{i}\right)$ is nonzero, then we have

$$
\begin{aligned}
A(s) & =\left(\frac{1}{p}\right)^{\ell_{1}(w) s+\ell_{2}(w)} \cdot \prod_{i=1}^{n} \sum_{a_{i} \in \mathbb{N}}\left(\frac{1}{p}\right)^{a_{i}\left(\ell_{1}\left(v_{i}\right) s+\ell_{2}\left(v_{i}\right)\right)} \\
& =\left(\frac{1}{p}\right)^{\ell_{1}(w) s+\ell_{2}(w)} \cdot \prod_{i=1}^{n} \frac{1}{1-\left(\frac{1}{p}\right)^{\ell_{1}\left(v_{i}\right) s+\ell_{2}\left(v_{i}\right)}}
\end{aligned}
$$

The assertions in the lemma are direct consequences of this formula.

We can now give the proof of our result on Igusa-type zeta functions associated to cones.

Proof of Theorem 1.3. Arguing by induction on the dimension of $\sigma$, we may assume that the theorem holds for all cones of smaller dimension than $\operatorname{dim}(\sigma)$ (the case when $\operatorname{dim}(\sigma)$ is zero being trivial). In this case, we see that proving the assertions in the theorem for $Z_{\sigma, \ell_{1}, \ell_{2}}$ is equivalent with proving them for $\bar{Z}_{\sigma, \ell_{1}, \ell_{2}}$.

We first show that it is enough to prove the theorem when $\sigma$ is a simplicial cone. Indeed, it is well-known that one can always find a fan $\Gamma$ refining the cone $\sigma$ such that every cone in $\Gamma$ is simplicial and the one-dimensional cones in $\Gamma$ are precisely the rays of $\sigma$. Since

$$
\bar{Z}_{\sigma, \ell_{1}, \ell_{2}}=\sum_{\tau \in \Gamma} Z_{\tau, \ell_{1}, \ell_{2}}
$$

and since each ray of a cone in $\Gamma$ is a ray of $\sigma$, we see that it is enough to prove the theorem for each (maximal) cone in $\Gamma$. 
Therefore we may assume that $\sigma$ is simplicial and our goal is to show that $\bar{Z}_{\sigma, \ell_{1}, \ell_{2}}$ satisfies the assertions in the theorem. Let $v_{1}, \ldots, v_{r}$ be the primitive generators of the rays of $\sigma$. Since $\sigma$ is simplicial, the $v_{i}$ are linearly independent over $\mathbb{Q}$. The semigroup $\sigma \cap N$ is finitely generated, so we may choose generators $w_{1}, \ldots, w_{s}$. The $v_{i}$ span $\sigma$ over $\mathbb{Q}$, hence we can find a positive integer $m$ such that every $m w_{j}$ is in the semigroup generated by the $v_{i}$. It follows that after replacing $\left\{w_{1}, \ldots, w_{s}\right\}$ by $\left\{q_{1} w_{1}+\ldots+q_{s} w_{s} \mid 0 \leq q_{j} \leq m-1\right\}$, we may assume that

$$
\sigma \cap N=\bigcup_{j=1}^{s}\left(w_{j}+S\right),
$$

where $S$ is the semigroup generated by the $v_{i}$.

If $I \subseteq\{1, \ldots, s\}$, let us put

$$
A_{I}(s):=\sum_{v}\left(\frac{1}{p}\right)^{\ell_{1}(v) s+\ell_{2}(v)}
$$

where the sum is over $v$ in $\bigcap_{j \in I}\left(w_{j}+S\right)$. We claim that $\bigcap_{j \in I}\left(w_{j}+S\right)$ is either empty or it is equal to $w+S$ for a suitable $w$ in $N$. Indeed, by an obvious induction on $|I|$ it is enough to show this when $I$ has two elements, say $j$ and $k$. The intersection of $w_{j}+S$ and $w_{k}+S$ is nonempty if and only if $w_{j}-w_{k}$ lies in the lattice generated by the $v_{i}$. If this is the case, let us write $w_{j}-w_{k}=\sum_{i=1}^{r} a_{i} v_{i}$ for suitable integers $a_{1}, \ldots, a_{r}$. If we put $w=w_{j}+\sum_{i=1}^{r} \max \left\{0,-a_{i}\right\} v_{i}$, then it is easy to check that $\left(w_{j}+S\right) \cap\left(w_{k}+S\right)=w+S$, which proves our claim.

It follows from our claim and Lemma 3.1 that each $A_{I}$ is a rational function of $\left(\frac{1}{p}\right)^{s}$. Moreover, if $s$ is a pole of $A_{I}$, then there is $i$ such that $\operatorname{Re}(s)=-\frac{\ell_{2}\left(v_{i}\right)}{\ell_{1}\left(v_{i}\right)}$. On the other hand, it follows from (7) that

$$
\bar{Z}_{\sigma, \ell_{1}, \ell_{2}}=\sum_{I}(-1)^{|I|-1} A_{I}(s),
$$

where the sum is over all nonempty subsets $I$ of $\{1, \ldots, s\}$. Therefore $\bar{Z}_{\sigma, \ell_{1}, \ell_{2}}$ satisfies the assertions of the theorem, which completes the proof.

Putting together Theorem 1.3 and the description of the Igusa zeta function of a monomial ideal from the previous section, we can relate the poles of this zeta function with the torus-invariant divisors in the blowing-up along the ideal.

Proof of Theorem 1.4. It follows from Proposition 2.1 and Theorem 1.3 that if $s$ is a pole of $Z_{I}$, then there is a primitive generator $v$ of a ray of the normal fan $\Delta_{I}$ to the Newton polyhedron $P_{I}$ such that

$$
\operatorname{Re}(s)=-\frac{\langle e, v\rangle}{\langle w, v\rangle} .
$$

Here $w$ is a vertex of $P_{I}$ such that $v$ is contained in the maximal cone $\sigma_{w}$ of $\Delta_{I}$ corresponding to $w$.

On the other hand, recall that the torus-invariant divisors on the toric variety defined by $\Delta_{I}$ correspond precisely to the rays of $\Delta_{I}$. Moreover, if $E$ is the divisor corresponding to the ray through $v$, then $k_{E}=\langle e, v\rangle-1$. Since we also have

$$
a_{E}(I)=\min \left\{\langle u, v\rangle \mid x^{u} \in I\right\}=\langle w, v\rangle,
$$

as $v$ lies in $\sigma_{w}$, we deduce the assertion in the theorem. 


\section{Poles And roots of the Bernstein-Sato Polynomial}

We now show that the real part of any pole of $Z_{I}$ is a root of the Bernstein-Sato polynomial $b_{I}$ associated to $I$. In fact, we prove the following stronger statement that together with Theorem 1.4 implies Theorem 1.2.

Proposition 4.1. If $I$ is a nonzero proper monomial ideal and if $E$ is a prime divisor in the normalized blowing-up of the affine space along $I$ such that $a_{E}(I)$ is nonzero, then $-\frac{k_{E}+1}{a_{E}(I)}$ is a root of the Bernstein-Sato polynomial $b_{I}$.

Proof. The divisor $E$ corresponds to a ray in the normal fan $\Delta_{I}$ to $P_{I}$. Let $v$ be a primitive generator of this ray. If $w$ is a vertex of $P_{I}$ such that the corresponding maximal cone $\sigma_{w}$ of $\Delta_{I}$ contains $v$, then we have seen in the proof of Theorem 1.4 that $k_{E}+1=\langle e, v\rangle$ and $a_{E}(I)=\langle w, v\rangle$. Note that since $\langle w, v\rangle \neq 0$, the facet $Q$ of $P_{I}$ corresponding to $v$ is not contained in a coordinate hyperplane: if, for example, $Q$ is contained in the hyperplane $\left(x_{i}=0\right)$, then $v=e_{i}$, and since $w$ lies in $Q$ we get $\langle w, v\rangle=0$, a contradiction.

In order to show that $\left(k_{E}+1\right) / a_{E}(I)$ is a root of the Bernstein-Sato polynomial $b_{I}$ associated to $I$, we use the description of the roots of $b_{I}$ from BMS2] (in fact, the ones that we need for the theorem are "the most straightforward" of the roots of $\left.b_{I}\right)$. Since $Q$ is a facet of $P_{I}$ that is not contained in a coordinate hyperplane, there is a unique linear function $L_{Q}$ on $M_{\mathbb{R}}$ such that $Q=P_{I} \cap L_{Q}^{-1}(1)$. With this notation, it is shown in BMS2] (see Remark 4.6) that $-L_{Q}(e)$ is a root of $b_{I}$.

On the other hand, since the ray through $v$ corresponds to the facet $Q$ and since $w$ is in $Q$, we have

$$
Q=\left\{u \in P_{I} \mid\langle u, v\rangle=\langle w, v\rangle\right\}
$$

Therefore $L_{Q}$ is given by $\frac{1}{\langle w, v\rangle} \cdot v$, and since $-L_{Q}(e)$ is a root of $b_{I}$, we see that $\left(k_{E}+1\right) / a_{E}(I)$ is, indeed, a root of $b_{I}$.

Remark 4.2. We do not know whether the analogue of Proposition 4.1 holds for a nonnecessarily monomial ideal $I$. Note that if $I=(f)$ is principal, then the assertion is trivial: the divisor $E$ is one of the irreducible components of the divisor $H$ defined by $f, k_{E}=0$, and $a_{E}(I)$ is the multiplicity of $E$ in $H$. The fact that $-\frac{1}{a_{E}(f)}$ is a root of $b_{f}$ then follows by restricting to an open subset where $E$ is smooth and $H=a_{E}(f) \cdot E$.

Remark 4.3. The arguments in the previous two sections can also be used to analyze the orders of the possible poles of the Igusa zeta function $Z_{I}$. Indeed, it follows from Proposition 2.1 and from the proof of Theorem 1.3 that if $s$ is a pole of order $r$ of $Z_{I}$, then $r \leq n$ and there are $r$ invariant divisors $E_{1}, \ldots, E_{r}$ on the normalized blowing-up along $I$ such that $E_{1} \cap \ldots \cap E_{r} \neq \emptyset$ and $\operatorname{Re}(s)=-\left(k_{E_{i}}+1\right) / a_{E_{i}}(I)$ for every $i$. We would like to deduce that in this case $\operatorname{Re}(s)$ is a root of order $r$ of $b_{I}$, but unfortunately, we do not understand well enough the multiplicities of the roots of $b_{I}$.

Remark 4.4. While Proposition 2.1 gives in principle a formula for the Igusa zeta function of a monomial ideal, and Theorem 1.4 gives an estimate on the denominator of this function (written as a rational function of $1 / p^{s}$ ), getting a general explicit formula for the denominator is rather difficult. A Maple code for computing $p$ adic and motivic zeta functions of monomial ideals via resolution of singularities is available, upon request, from the first author. 
Remark 4.5. Using motivic integration, Denef and Loeser defined in [DL] a motivic analogue of the Igusa zeta function. For concreteness, we preferred to work with $p$ adic integrals. However, as the reader familiar with this topic will certainly notice, all the above results have analogues in the motivic setting, "replacing $p$ by $\mathbb{L}$ ". For example, if $\sigma, \ell_{1}$ and $\ell_{2}$ are as in Theorem 1.3, then the series

$$
\sum_{v \in \sigma \cap N} \mathbb{L}^{-\left(\ell_{1}(v) s+\ell_{2}(v)\right)}
$$

can be written as a sum of fractions with numerator in $K\left[\mathbb{L}^{-s}\right]$ and denominator of the form

$$
\prod_{i=1}^{r}\left(1-\mathbb{L}^{-\left(\ell_{1}\left(v_{i}\right) s+\ell_{2}\left(v_{i}\right)\right)}\right)
$$

where $r \leq \operatorname{dim}(\sigma)$ and $v_{1}, \ldots, v_{r}$ are primitive generators of the rays of $\sigma$. Here $K$ is the ring obtained from the Grothendieck ring of varieties over a base field $k$ by inverting $\mathbb{L}=\left[\mathbb{A}_{k}^{1}\right]$. Similarly, if $I$ is a monomial ideal, then the motivic zeta function of $I$

$$
\int_{\left(\mathbb{A}^{n}\right)_{\infty}} \mathbb{L}^{-s \cdot \operatorname{ord}_{t} I}
$$

can be written as a sum of fractions with numerator in $K\left[\mathbb{L}^{-s}\right]$ and denominator of the form

$$
\prod_{i=1}^{r}\left(1-\mathbb{L}^{-\left(a_{E_{i}}(I) s+k_{E_{i}}+1\right)}\right),
$$

where $r \leq n$ and $E_{1}, \ldots, E_{r}$ are divisors on the normalized blowing-up of $\mathbb{A}^{n}$ along $I$ such that $E_{1} \cap \ldots \cap E_{r}$ is nonempty.

\section{ACKNOWLEDGMEnT}

The second author would like to thank Wim Veys for a very inspiring discussion on Igusa zeta functions.

\section{REFERENCES}

[Be] J. N. Bernstein, Analytic continuation of generalized functions with respect to a parameter, Funk. Anal. 6 (1972), 26-40. MR0320735 (47:9269)

[BMS1] N. Budur, M. Mustaţă and M. Saito, Combinatorial description of the roots of the Bernstein-Sato polynomials for monomial ideals, Comm. Algebra. 34 (2006), 4103-4117. MR2267574 (2007h:32041)

[BMS2] N. Budur, M. Mustaţă and M. Saito, Roots of Bernstein-Sato polynomials for monomial ideals: a positive characteristic approach, Math. Res. Lett. 13 (2006), 125-142. MR2200051(2006k:14003)

[BMS3] N. Budur, M. Mustaţă and M. Saito, Bernstein-Sato polynomials of arbitrary varieties, Compos. Math. 142 (2006), 779-797. MR2231202 (2007c:32036)

[De] J. Denef, Report on Igusa's local zeta function, Séminaire Bourbaki, Vol. 1990/91, Astérisque No. 201-203 (1991), Exp. No. 741, 359-386. MR.1157848 (93g:11119)

[DH] J. Denef and K. Hoornaert, Newton polyhedra and Igusa's local zeta function, J. Number Theory 89 (2001), 31-64. MR.1838703 (2002g:11170)

[DL] J. Denef and F. Loeser, Motivic Igusa zeta functions, J. Algebraic Geom. 7 (1998), 505537. MR 1618144 (99j:14021)

[Fu] W. Fulton, Introduction to toric varieties, Annals of Mathematics Studies 131, The William H. Roever Lectures in Geometry, Princeton University Press, Princeton, NJ, 1993. MR 1234037 (94g:14028) 
[Ig] J.-i. Igusa, An introduction to the theory of local zeta functions, AMS/IP Studies in Advanced Mathematics, 14, American Mathematical Society, Providence, RI; International Press, Cambridge, MA, 2000. MR.1743467 (2001j:11112)

[Lo] F. Loeser, Fonctions d'Igusa $p$-adiques, polynômes de Bernstein, et polyhédres de Newton, J. Reine Angew. Math. 412 (1990), 75-96. MR1079002 (92c:11139)

[SS] M. Sato and T. Shintani, On zeta functions associated with prehomogeneous vector spaces, Proc. Nat. Acad. Sci. U.S.A. 69 (1972), 1081-1082. MR0296079 (45:5140)

[Ve] W. Veys, Embedded resolution of singularities and Igusa's local zeta function, Academiae Analecta (2001), available at wis.kuleuven.be/algebra/veys.htm.

[Zu] W. A. Zúñiga-Galindo, On the poles of the Igusa zeta function for algebraic sets, Bull. London Math. Soc. 36 (2004), 310-320. MR2038719 (2005c:11149)

Department of Mathematics and Computer Science, John Carroll University, 20700 North Park Blvd., University Heights, Ohio 44118

Current address: Department of Mathematics, SUNY Potsdam, 44 Pierrepont Avenue, Potsdam, New York 13676-2294

E-mail address: howaldja@potsdam.edu

Department of Mathematics, University of Michigan, Ann Arbor, Michigan 48109

E-mail address: mmustata@umich.edu

Department of Mathematics, University of Michigan, Ann Arbor, Michigan 48109 Current address: Department of Mathematics, University of Kentucky, 825 Patterson Office Tower, Lexington, Kentucky 40506

E-mail address: cyuen@ms.uky.edu 